\title{
Insetos aquáticos e seus grupos tróficos relacionados a cobertura vegetal de propriedades leiteiras no Vale do Taquari/RS
}

Esta pesquisa teve como objetivo identificar os grupos tróficos funcionais das famílias de insetos aquáticos encontrados em 12 propriedades leiteiras classificadas conforme sua cobertura vegetal, localizadas na microbacia hidrográfica do Arroio da Seca, porção Taquari-Antas. As amostragens foram realizadas de fevereiro a novembro do ano de 2019 ao longo das margens d'água de arroios de cada propriedade. Foram coletados e identificados um total de 2.741 indivíduos de insetos aquáticos, pertencentes a 8 ordens e 38 famílias. Não houve diferença significativa entre a riqueza e a abundância dos grupos tróficos funcionais de insetos aquáticos entre os pontos de coleta, porém, na composição de famílias houve diferença. $O$ estudo revela que as condições ambientais das propriedades leiteiras, em relação à cobertura vegetal em suas Áreas de Preservação Permanente, possuem influência na determinação da estrutura e composição da comunidade da entomofauna aquática.

Palavras-chave: Bioindicadores; Entomofauna aquática; Pecuária leiteira; Sustentabilidade.

\section{Aquatic insects and their trophic groups related to plant cover of dairy properties in Vale do Taquari/RS}

\begin{abstract}
This research aimed to identify the trophic groups supplied from the families of aquatic insects found in 12 dairy farms classified according to their vegetation cover, obligatorily in the hydrographic watershed of Arroio da Seca, Taquari-Antas portion. Sampling was carried out from February to November 2019 , along the banks of streams of each property. A total of 2,741 belonging to aquatic insects were collected and identified, belonging to 8 orders and 38 families. There was no significant difference between the richness and abundance of the proportional trophic groups of aquatic insects between the collection points, however, in the composition of families there was no difference. The study reveals that the environmental conditions of farms, in relation to vegetation cover in their Permanent Preservation Areas, have an influence on determining the structure and composition of the aquatic entomofauna community.
\end{abstract}

Keywords: Bioindicators; Aquatic entomofauna; Dairy farming; Sustainability.

Topic: Conservação da Biodiversidade

Reviewed anonymously in the process of blind peer.
Received: 04/03/2021

Approved: 23/03/2021
Bruna Ehlert (ii)

Universidade do Vale do Taquari, Brasil http://lattes.cnpq.br/6461716175156655 http://orcid.org/0000-0003-3308-2914 bruna.ehlert@hotmail.com

\section{Claudete Rempel (id}

Universidade do Vale do Taquari, Brasil http://lattes.cnpq.br/8340497822227462

http://orcid.org/0000-0001-8573-0237

crempel@univates.br

\section{Marina Schmidt Dalzochio (iD)}

Universidade do Vale do Rio dos Sinos, Brasil

http://lattes.cnpq.br/8400882066099257

http://orcid.org/0000-0001-9241-921X

mahsdalzochio@gmail.com

\author{
Patrícia Caye Bergmann (iD \\ Universidade do Vale do Taquari, Brasil \\ http://lattes.cnpq.br/8497886339173527 \\ http://orcid.org/0000-0003-0081-9158 \\ patricia.bergmann@universo.univates.br \\ Cristian Mateus Zerwes (id \\ Universidade do Vale do Taquari, Brasil \\ http://lattes.cnpq.br/9316007567169561 \\ http://orcid.org/0000-0002-1503-2780 \\ cristianzerwes@universo.univates.br
}

Referencing this:

EHLERT, B.; REMPEL, C.; DALZOCHIO, M. S.; BERGMANN, P. C.;

ZERWES, C. M.. Insetos aquáticos e seus grupos tróficos relacionados a cobertura vegetal de propriedades leiteiras no Vale do Taquari/RS. Revista Ibero Americana de Ciências Ambientais, v.12, n.3, p.102110, 2021. DOI: http://doi.org/10.6008/CBPC21796858.2021.003.0010 


\section{INTRODUÇÃO}

Diversas atividades antrópicas comprometem a qualidade ambiental dos recursos hídricos, muitas dessas relacionadas à agricultura (SABATER et al., 2018). Dentre tais atividades destaca-se a pecuária leiteira, sendo o Brasil é o quarto maior produtor mundial de leite de vaca (BRASIL, 2017) e o estado do Rio Grande do Sul o terceiro maior produtor nacional, onde o Vale do Taquari destaca-se por ser a terceira região de maior produção leiteira do estado.

A atividade leiteira pode provocar efeitos prejudiciais na qualidade da água, na sua biota e consequentemente no seu funcionamento (HUGHES et al., 2016; SCANES, 2018). Entre os organismos presentes nos ambientes lóticos, estão os insetos aquáticos, os quais possuem a sua distribuição associada a diferentes fatores, como a sensibilidade frente aos níveis de poluição, aos hábitats disponíveis, interações bióticas e disponibilidade de alimentos (JOLY et al., 1998; ESTEVES, 2011).

A entomofauna aquática pode ser classificada em diferentes Grupos Tróficos Funcionais (GTF), de acordo como adquirem seus recursos alimentares, como: Fragmentadores, Coletores, Raspadores, Predadores, Catadores e Filtradores, alguns táxons podem ser considerados "Generalistas", pois abrangem várias guildas alimentares, consumindo uma ampla gama de recursos (MERRITT et al., 1984; CHAGAS et al., 2017; HENTGES, 2019). O conhecimento sobre os GTF dos insetos aquáticos fornece informações úteis sobre a estabilidade do ecossistema e permite avaliar a resposta desses organismos a variáveis ambientais (KIKUCHI et al., 1998, MERRITT et al., 2017).

Neste contexto, o objetivo deste estudo foi de identificar o GTF das famílias de insetos aquáticos associadas às APP dos ambientes lóticos de propriedades leiteiras que possuem suas áreas de cobertura vegetal classificadas de acordo com a proposta sugerida por Markus (2014), relacionada ao Subparâmetro de diversidade da Mata Nativa na APP onde a fitossociologia e a florística são os indicadores de sustentabilidade ambiental.

\section{MATERIAIS E MÉTODOS}

O estudo foi realizado na microbacia do Arroio da Seca um afluente da margem esquerda do Rio Taquari, nos municípios de Imigrante e Colinas, Estado do Rio Grande do Sul, Brasil. A presente pesquisa desenvolve-se no âmbito do Grupo de Pesquisa em Avaliação da Sustentabilidade de Propriedades Produtoras de Leite e com registro no SISBIO número 73016-1.

A vegetação da região é classificada como Floresta Estacional Decidual e o clima é subtropical (KÖPPEN, 1931) com temperatura média anual de 18 C aproximadamente e precipitação média variando entre 1.300 e $1.800 \mathrm{~mm}$ anualmente, segundo o INPE. Um estudo com uma descrição detalhada da vegetação ocorrente nestes locais foi apresentado por Zerwes et al. (2020), que analisou o estrato arbóreo de fragmentos florestais de formação submontana nas áreas de APP dos ambientes lóticos, onde através do levantamento de campo foram calculados índices fitossociológicos e de diversidade (TABELA 1). A partir dos resultados de Zerwes (2015) aplicamos nesta pesquisa a proposta sugerida por Markus (2014) relacionada 
ao Sub-parâmetro de diversidade da Mata Nativa na APP, de acordo com os índices de diversidade de Shannon, onde a fitossociologia e a florística são os indicadores de sustentabilidade ambiental (TABELA 1). Para a coleta dos insetos aquáticos, foi amostrado um ponto específico as margens dos ambientes lóticos de cada uma das propriedades pesquisadas por Zerwes (2015) (TABELA 1), citado anteriormente, sendo no total doze propriedades, duas dessas pertencem ao município de Colinas, e dez no município de Imigrante.

Tabela 1: Localização dos pontos amostrados no estudo e o Índice de Shannon encontrado por Zerwes (2015) em cada propriedade, com a referida classificação proposta por Markus (2014).

\begin{tabular}{|c|c|c|c|c|}
\hline \multirow{4}{*}{$\begin{array}{l}\text { Propriedades } \\
\text { leiteiras } \\
\text { P1 }\end{array}$} & \multicolumn{2}{|l|}{$\begin{array}{l}\text { Coordenadas } \\
\text { geográficas }\end{array}$} & \multirow[t]{2}{*}{$\begin{array}{l}\text { Índice de Shannon do estudo } \\
\text { de Zerwes (2015) }\end{array}$} & \multirow{2}{*}{$\begin{array}{l}\text { Conceito das propriedades, de acordo com o Índice de } \\
\text { Shannon proposto por Markus (2014) } \\
\text { Excelente }\end{array}$} \\
\hline & \multicolumn{2}{|c|}{$29^{\circ} 21^{\prime} 44,8^{\prime \prime} \mathrm{W}$} & & \\
\hline & $51^{\circ} 47^{\prime} 59,3^{\prime \prime}$ & & \multirow[t]{2}{*}{3,52} & \\
\hline & S $\quad 29^{\circ} 19^{\prime} 19,91^{\prime \prime}$ & w & & Excelente \\
\hline \multirow[t]{2}{*}{ P2 } & $51^{\circ} 45^{\prime} 10,79^{\prime \prime}$ & & 3,16 & \\
\hline & S $\quad 29^{\circ} 19^{\prime} 54,5^{\prime \prime}$ & w & & Excelente \\
\hline \multirow[t]{2}{*}{ P3 } & $51^{\circ} 45^{\prime} 17,4^{\prime \prime}$ & & 3,52 & \\
\hline & S $\quad 29^{\circ} 21^{\prime} 02,97^{\prime \prime}$ & w & & Excelente \\
\hline \multirow[t]{2}{*}{ P4 } & $51^{\circ} 47^{\prime} 28,74^{\prime \prime}$ & & 3,06 & \\
\hline & S29²3'29,5" & & & Excelente \\
\hline \multirow[t]{2}{*}{ P5 } & W51 $49^{\circ} 59,35^{\prime \prime}$ & & 3,24 & \\
\hline & S29²3'32.51" & & & Excelente \\
\hline \multirow[t]{2}{*}{ P6 } & W5150'43.62" & & 3,48 & \\
\hline & S $\quad 29^{\circ} 20^{\prime} 34,3^{\prime \prime}$ & w & & Excelente \\
\hline \multirow[t]{2}{*}{ P7 } & $51^{\circ} 45^{\prime} 34,2^{\prime \prime}$ & & 3,66 & \\
\hline & S $\quad 29^{\circ} 21^{\prime} 47,4^{\prime \prime}$ & w & & Excelente \\
\hline \multirow[t]{2}{*}{ P8 } & $51^{\circ} 45^{\prime} 25,9^{\prime \prime}$ & & 3,56 & \\
\hline & S $29^{\circ} 19^{\prime} 40,23^{\prime \prime}$ & w & & Excelente \\
\hline \multirow[t]{2}{*}{ P9 } & $51^{\circ} 43^{\prime} 31,95^{\prime \prime}$ & & 3,48 & \\
\hline & S $\quad 29^{\circ} 22^{\prime} 00,9^{\prime \prime}$ & w & & Bom \\
\hline \multirow[t]{2}{*}{ P10 } & $51^{\circ} 51^{\prime} 09,6^{\prime \prime}$ & & 2,79 & \\
\hline & S $\quad 29^{\circ} 32^{\prime} 28,5^{\prime \prime}$ & w & & Excelente \\
\hline \multirow[t]{2}{*}{ P11 } & $51^{\circ} 49^{\prime} 59,0^{\prime \prime}$ & & 3,43 & \\
\hline & S $\quad 29^{\circ} 20^{\prime} 3$, & & & Excelente \\
\hline P12 & $51^{\circ} 46^{\prime} 13,2^{\prime \prime}$ & & 3,50 & \\
\hline
\end{tabular}

Fonte: Adaptada de Markus (2014); Zerwes (2015).

\section{Área de estudo}

A Bacia Hidrográfica do Rio Taquari-Antas situa-se na região nordeste do Estado do Rio Grande do Sul e nasce no extremo leste da Bacia com a denominação de Rio das Antas até a foz do Rio Carreiro, quando passa a denominar-se Taquari, desembocando no Rio Jacuí. Esta Bacia é composta por rios de primeira, segunda e terceira ordem e possui uma extensão $546 \mathrm{Km}$ desde a nascente até a sua foz, sendo que $359 \mathrm{~km}$ correspondem ao Rio das Antas e 187 km ao Rio Taquari.

Os locais avaliados neste estudo fazem parte da porção Taquari e estão situados na microbacia hidrográfica do Arroio da Seca que abrange uma área de aproximadamente 13.868 hectares. Na microbacia do Arroio da Seca localizam-se os municípios de Imigrante, Colinas, Roca Sales, Teutônia, Westfália, Coronel Pilar, Boa Vista do Sul, Garibaldi e Carlos Barbosa (IBGE, 2014). A vegetação da região é classificada como Floresta Estacional Decidual e o clima é subtropical (KÖPPEN, 1931) com temperatura média anual de $18 \mathrm{C}$ aproximadamente e precipitação média de 1.600 mm (NIH, 2012).

A presente pesquisa desenvolve-se no âmbito do Grupo de Pesquisa em Avaliação da Sustentabilidade de Propriedades Produtoras de Leite e com registro no SISBIO número 73016-1. Foram 
avaliadas 12 propriedades leiteiras para a coleta dos insetos aquáticos, duas dessas pertencem ao município de Colinas, e as demais ao município de Imigrante.

\section{Amostragem dos insetos aquáticos}

Para a captura dos insetos aquáticos foram realizadas um total de quatro coletas nas margens d'água de cada propriedade leiteira, nos meses de fevereiro, maio, agosto e novembro. Os insetos aquáticos foram coletados com uma amostrador Surber, conforme a metodologia adaptada de Strohschoen et al. (2009), num total de $4 \mathrm{~m}^{2}$, compostos por 4 réplicas de $1 \mathrm{~m}^{2}$ de trecho amostrado por ponto, com um esforço amostral 30 minutos nas margens d'água da sede da propriedade. As quatro amostras obtidas no local foram integralizadas para compor um ponto de coleta, embora acondicionadas e processadas individualmente.

O material coletado foi acondicionado em frascos contendo álcool etílico $70 \%$, em seguida todo material biológico foi separado do material orgânico em uma placa de Petri sob iluminação e após identificados, com auxílio de uma lupa estereoscópica até o nível de família, utilizando chaves dicotômicas específicas (MERRIT et al., 1984; LOPRETTO et al., 1995; FERNÁNDEZ et al., 2001; PES et al., 2005; BENETTI et al., 2006; COSTA et al., 2006; MUGNAl et al., 2010; HAMADA et al., 2014). Após a identificação os espécimes foram colocados em frascos identificados contendo álcool a 70\%. O material será depositado na coleção entomológica do Museu de Ciências Naturais da Univates (MCNU).

\section{Análises de dados}

Os insetos aquáticos coletados foram categorizados de acordo com seus respectivos Grupos Tróficos Funcionais (GTF) através de bibliografias especializadas (CUMMINS et al., 1979; MERRIT et al., 1984) (QUADRO 2). A análise funcional foi realizada por meio do software $\mathrm{R}$ com auxílio do pacote estatístico FD e da função dbFD para associar a matriz de grupos tróficos funcionais e a abundância dos organismos. Como as famílias poderiam apresentar mais de um grupo trófico, essas foram classificadas em 0 e 1 e submetidas à análise para criar apenas uma matriz de composição trófica, de acordo com a sua abundância.

Para comparar as diferenças de abundância e riqueza funcional dos insetos aquáticos entre os pontos amostrados foi empregado o Teste de Friedman, o qual comparou os mesmos meses amostrados entre os pontos de coleta. Foi realizado uma Análise de Variância Multivariada Permutacional - PERMANOVA de um fator, com similaridade de Bray-curtis e 9999 permutações para avaliar se haviam diferenças na composição de famílias dos diferentes grupos funcionais entre os pontos amostrados. Para visualizar os resultados da PERMANOVA foi utilizado como método de ordenação o escalonamento multidimensional não métrico (NMDS). As análises estatísticas foram realizadas no software PAST 4.02.

\section{RESULTADOS}

Foram encontrados 2.741 indivíduos, estes foram identificados em 38 famílias de insetos aquáticos e estes foram categorizados conforme seu GTF em: Fragmentadores, Predadores, Catadores, Raspadores, Filtradores e Generalistas (QUADRO 1). 
Quadro 1: Abundância das famílias de insetos aquáticos presentes nos pontos do estudo e seus respectivos Grupos Tróficos Funcionais.

\begin{tabular}{|c|c|c|c|c|c|c|c|c|c|c|c|c|}
\hline GRUPO TRÓFICO FUNCIONAL/FAMÍLIAS & P1 & P2 & P3 & P4 & P5 & P6 & P7 & P8 & P9 & P10 & P11 & P12 \\
\hline \multicolumn{13}{|l|}{ CATADORES } \\
\hline Hydrophilidae & 0 & 0 & 0 & 1 & 0 & 0 & 0 & 0 & 0 & 0 & 10 & 0 \\
\hline Leptohyphidae & 63 & 6 & 0 & 8 & 2 & 2 & 27 & 20 & 63 & 18 & 21 & 4 \\
\hline Caenidae & 7 & 27 & 6 & 6 & 10 & 12 & 13 & 17 & 25 & 2 & 234 & 63 \\
\hline Baetidae & 5 & 3 & 11 & 2 & 2 & 0 & 3 & 1 & 3 & 2 & 13 & 1 \\
\hline \multicolumn{13}{|l|}{ PREDADORES } \\
\hline Perlidae & 0 & 6 & 11 & 1 & 3 & 0 & 1 & 1 & 1 & 1 & 0 & 3 \\
\hline Dytiscidae & 0 & 0 & 0 & 0 & 0 & 0 & 0 & 0 & 0 & 0 & 0 & 1 \\
\hline Tipulidae & 0 & 2 & 0 & 1 & 1 & 0 & 1 & 1 & 0 & 1 & 0 & 3 \\
\hline Tabanidae & 5 & 3 & 0 & 1 & 1 & 0 & 3 & 3 & 1 & 1 & 21 & 2 \\
\hline Empididae & 0 & 0 & 0 & 0 & 0 & 1 & 0 & 0 & 0 & 0 & 0 & 0 \\
\hline Libellulidae & 6 & 0 & 0 & 1 & 0 & 1 & 2 & 1 & 5 & 4 & 44 & 1 \\
\hline Gomphidae & 40 & 1 & 0 & 0 & 0 & 1 & 2 & 1 & 4 & 7 & 1 & 0 \\
\hline Calopterygidae & 1 & 2 & 0 & 0 & 2 & 2 & 2 & 0 & 1 & 0 & 0 & 0 \\
\hline Coenagrionidae & 0 & 0 & 0 & 0 & 0 & 7 & 0 & 0 & 1 & 1 & 36 & 1 \\
\hline Aeshnidae & 0 & 0 & 0 & 0 & 0 & 3 & 0 & 0 & 0 & 0 & 3 & 0 \\
\hline Veliidae & 1 & 16 & 0 & 2 & 5 & 0 & 14 & 3 & 0 & 0 & 0 & 43 \\
\hline Naucoridae & 0 & 0 & 0 & 0 & 0 & 0 & 0 & 0 & 0 & 1 & 1 & 0 \\
\hline Belostomatidae & 0 & 0 & 0 & 0 & 0 & 0 & 0 & 1 & 0 & 1 & 5 & 0 \\
\hline Gelastocoridae & 0 & 0 & 0 & 0 & 0 & 0 & 0 & 0 & 0 & 0 & 1 & 0 \\
\hline Mesoveliidae & 0 & 0 & 0 & 0 & 0 & 0 & 0 & 0 & 1 & 0 & 1 & 0 \\
\hline Gerridae & 0 & 0 & 0 & 0 & 0 & 0 & 0 & 0 & 0 & 0 & 0 & 1 \\
\hline \multicolumn{13}{|l|}{ RASPADORES } \\
\hline Elmidae & 4 & 29 & 77 & 9 & 3 & 23 & 20 & 2 & 9 & 2 & 4 & 10 \\
\hline Psephenidae & 2 & 2 & 13 & 1 & 4 & 0 & 1 & 20 & 0 & 11 & 36 & 2 \\
\hline Glossosomatidae & 0 & 1 & 13 & 0 & 0 & 0 & 0 & 0 & 0 & 0 & 0 & 0 \\
\hline Hydroptilidae & 2 & 0 & 0 & 2 & 0 & 0 & 1 & 1 & 1 & 2 & 0 & 0 \\
\hline \multicolumn{13}{|l|}{ FRAGMENTADORES } \\
\hline Dryopidae & 0 & 0 & 0 & 0 & 0 & 0 & 1 & 1 & 0 & 0 & 0 & 0 \\
\hline Calamoceratidae & 6 & 2 & 2 & 2 & 1 & 9 & 4 & 13 & 3 & 0 & 12 & 6 \\
\hline Pyralidae & 0 & 0 & 1 & 1 & 0 & 0 & 1 & 0 & 0 & 0 & 0 & 0 \\
\hline Gripopterygidae & 0 & 0 & 0 & 0 & 0 & 0 & 0 & 0 & 0 & 1 & 0 & 0 \\
\hline \multicolumn{13}{|l|}{ GENERALISTAS } \\
\hline Chironomidae & 54 & 69 & 74 & 64 & 12 & 57 & 63 & 61 & 274 & 11 & 123 & 22 \\
\hline Leptophlebiidae & 0 & 9 & 6 & 6 & 2 & 2 & 1 & 4 & 0 & 11 & 4 & 1 \\
\hline \multicolumn{13}{|l|}{ FILTRADORES } \\
\hline Simuliidae & 0 & 0 & 1 & 0 & 0 & 1 & 3 & 0 & 0 & 0 & 0 & 0 \\
\hline Culicidae & 0 & 38 & 0 & 0 & 0 & 0 & 0 & 0 & 0 & 0 & 38 & 0 \\
\hline Hydropsychidae & 0 & 0 & 2 & 1 & 0 & 3 & 3 & 4 & 0 & 0 & 6 & 0 \\
\hline Polycentropodidae & 0 & 1 & 0 & 1 & 0 & 1 & 0 & 0 & 0 & 0 & 1 & 0 \\
\hline \multicolumn{13}{|l|}{$\begin{array}{l}\text { RASPADORES } \\
\text { FILTRADORES } \\
\text { CATADORES }\end{array}$} \\
\hline Psychodidae & 0 & 0 & 0 & 0 & 0 & 0 & 0 & 1 & 0 & 0 & 0 & 0 \\
\hline Leptoceridae & 121 & 22 & 3 & 10 & 5 & 0 & 6 & 11 & 8 & 7 & 23 & 20 \\
\hline
\end{tabular}

De acordo com a classificação dos GTF, os Generalistas foi o grupo com a maior abundância de organismos no estudo, devido à alta expressividade da família Chironomidae nos pontos amostrados. Em sete propriedades do estudo os Generalistas foram os que apresentaram maior destaque (P2, P4, P5, P6, P7, P8 e P9). Na propriedade do ponto 1 (P1) o maior número de organismos foi representado pela família Leptoceridae, que ocupa mais de um GTF, onde seus representantes são considerados Filtradores, Raspadores e Coletores. O ponto 3 (P3) apresentou um maior número de organismos Raspadores. No ponto 10 (P10) houve abundância equivalente de indivíduos Catadores e Generalistas, já nos pontos 11 (P11) e 12 (P12) o GTF dos Catadores foi o mais expressivo. Em relação à riqueza o GTF dos predadores foi o que 
apresentou o maior número de famílias.

A abundância dos GTF dos insetos aquáticos não variou entre os pontos de coleta ( $\mathbf{l}^{2}=15,51, \mathrm{gl}=11$, $p=0.15)$, assim como a riqueza não foi significativa entre os sítios amostrados $\left(\mathbb{Q}^{2}=11.379, g l=11, p=0.29\right.$ ) (FIGURA 1). Em relação a composição de famílias de insetos aquáticos entre os pontos amostrados a PERMANOVA indicou variação significativa ( $F=1.586, p=0.0002)$, entre os pontos: $P 1$ e P3 ( $p=0.02)$; P1 e P5 $(p=0.03) ; P 1$ e P10 ( $p=0.05) ; P 2$ e P5 ( $p=0.02) ;$ P3 e P10 ( $p=0.05) ;$ P3 e P11 ( $p=0.03) ;$ P3 e P12 ( $p=0.05) ; P 4$ e P11 ( $p=0.02) ;$ P5 e P11 ( $p=0.02) ; P 10$ e P11 ( $p=0.02)$ (FIGURA 2).

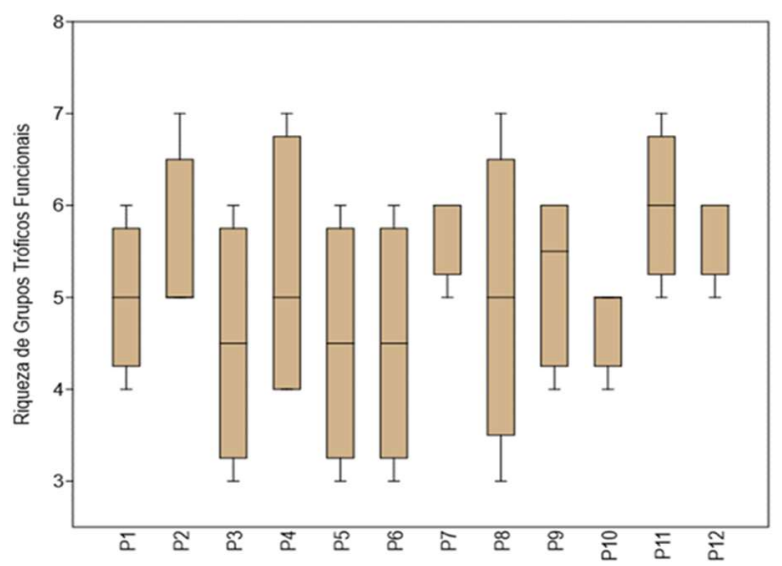

Figura 1: Riqueza dos GTF de insetos aquáticos nos doze pontos do estudo.

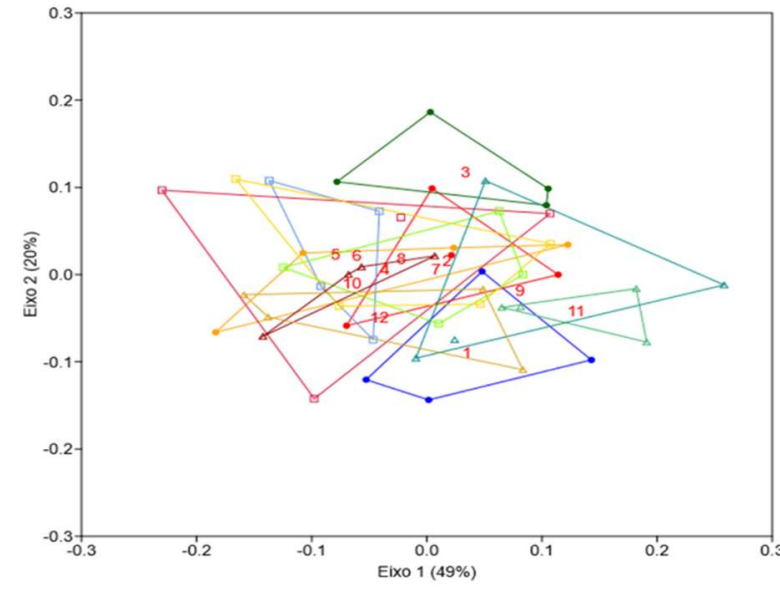

Figura 2: Escalonamento multidimensional não-métrico (NMDS) nos diferentes pontos do estudo e as famílias de insetos aquáticos.

\section{DISCUSSÃO}

As propriedades amostradas apresentaram 38 famílias de insetos aquáticos, representantes dos mais variados GTF em seus sítios de coleta, estes resultados demonstram as possíveis diferenças nos tipos de hábitats, sendo que a presença de diferentes grupos funcionais podem evidenciar uma diversidade de nichos tróficos existentes, de acordo com Copatti et al. (2010) quando distintos grupos de organismos exploram os mais variados hábitats, estes reduzem os efeitos da competição nos ambientes em que estão inseridos.

O grupo funcional com o maior número de famílias foi representado pelos predadores, este resultado pode estar relacionado à disponibilidade de presas presentes nestes ambientes (GIEHL, 2018). Nossos resultados corroboram pesquisas realizadas em ambientes lóticos que encontraram uma maior riqueza de macroinvertebrados predadores (HENTGES, 2019; NEGRÃO et al., 2019; PIO et al., 2020).

Em relação a abundância, os Generalistas apresentaram uma maior expressividade do estudo, devido a dominância da família Chironomidae em sete pontos amostrais. A abundância do táxon Chironomidae é muito comum em ambientes aquáticos devido sua alta taxa de crescimento populacional, como também aos seus diferentes hábitos alimentares (RIBEIRO et al., 2005; BIASI et al., 2013; CHOUDHARY et al., 2015; LESZCZYŃSKA et al., 2019). Estes táxons são considerados muito importantes para o funcionamento da cadeia alimentar nos ecossistemas aquáticos e na decomposição da matéria orgânica (COLLIER et al., 2009; SERRA et al., 2017; ONO et al., 2020). 
No ponto 1 (P1) a maior abundância de organismos foi representada pela família Leptoceridae que pertence ao GTF dos Raspadores, Catadores e Filtradores, indicando a alta oferta provinda da vegetação ciliar deste local, pois estes organismos possuem uma forte dependência de fontes de alóctones (RÍOS-TOUMA et al., 2011). O GTF Raspador apresentou uma maior abundância no ponto 3 (P3) indicando uma alta quantidade de material orgânico cedido pela vegetação ripária do entorno, no estudo de um autor em 2002 os ambientes mais preservados apresentaram maior abundância deste grupo trófico. 0 ponto 10 (P10) apresentou a mesma abundância de táxons Catadores e Generalistas e os pontos 11 (P11) e 12 (P12) organismos Catadores, de acordo com Hentges (2019) a presença de organismos Catadores é indicativa de matéria orgânica particulada em suspensão e depositada no fundo nos ecossistemas aquáticos.

A composição da comunidade de invertebrados diferiu entre os pontos amostrados, isso pode ser explicado pela ausência de algumas famílias e seus respectivos GTF em alguns locais de coleta, embora isso não tenha sido refletido estatisticamente numa menor riqueza e abundância no estudo. Nossos resultados estão de acordo com estudos que constataram diferença na composição de famílias de invertebrados em áreas com atividades agrícolas (CASTRO et al., 2016; IÑIGUEZ-ARMIJOS et al., 2018; FONTANA et al., 2020).

No presente estudo de todas as propriedades leiteiras classificadas conforme o conceito proposto por Markus (2014) apenas uma foi classificada como Boa (P10) e todas as demais como Excelentes, estas apresentaram famílias do grupo EPT (Ephemeteroptera, Plecoptera e Trichoptera), pertencentes a diferentes GTF, onde algumas famílias são consideradas sensíveis a alterações ambientais, mesmo na propriedade considerada "Boa" duas famílias representantes da ordem Plecoptera estiveram presentes, de dois diferentes GTF Filtrador (Perlidae) e Fragmentador (Gripopterygidae). Nossos resultados corroboram o estudo de Siegloch et al. (2016) que encontraram indivíduos sensíveis a poluição, pertencentes a variados GTF em ambientes aquáticos de maior complexidade de vegetação nativa.

\section{CONCLUSÕES}

As propriedades leiteiras analisadas apresentaram pouca variabilidade vegetal, sendo este um fato limitante neste estudo. Táxons de insetos aquáticos pertencentes aos diferentes GTF foram encontrados nos locais amostrados, demonstrando a variada oferta de hábitats, muitos destes proporcionados pela diversidade da cobertura vegetal presente em suas APP. Nesse sentido fica evidente a importância da preservação da vegetação ribeirinha nestes locais.

Para tanto, estudos complementares devem ser realizados com estes organismos ao longo dos pontos pesquisados, com o objetivo de promover a conservação dos recursos hídricos, através da identificação de fatores que podem alterar a qualidade da água dos recursos hídricos devido a produção da pecuária leiteira.

\section{AGRADECIMENTOS}

Aos bolsistas do grupo de pesquisa; à Coordenação de Aperfeiçoamento de Pessoal de Nível Superior, CAPES, Brasil. 


\section{REFERÊNCIAS}

BIASI, C.. Macroinvertebrados associados às folhas em decomposição de riachos neotropicais: influência da qualidade química, variedade de espécies vegetais, biomassa de fungos e tempo de exposição. Dissertação (Mestrado) Universidade Federal de Santa Maria, Santa Maria, 2013

BRASIL. Ministério da Agricultura, Pecuária e Abastecimento Secretaria de Política Agrícola. Brasil Projeções do Agronegócio 2016/2017 a 2026/2027. 8 ed. Brasília: MAPA, 2017.

CASTRO, D. M. P.; CARVALHO, D. R.; POMPEU, P. D. S.; MOREIRA, M. Z.; NARDOTO, G. B.; CALLISTO, M.. Land use influences niche size and the assimilation of resources by benthic macroinvertebrates in tropical headwater streams. PLos ONE, v.11, e0150527, 2016.

CHAGAS, F. B.; RUTKOSKI, C. F.; BIENIEK, G. B.; VARGAS, G. D. L. P.; HARTMANN, P. L.; HARTMANN, M. T.. Utilização da estrutura de comunidades de macroinvertebrados bentônicos como indicador de qualidade da água em rios no sul do Brasil. Revista Ambiente \& Água, v.12, n.3, 2017.

COLLIER, K. J.; HAMER, M.; CHADDERTON, L.. A new substrate for sampling deep river macroinvertebrates. New Zealand Natural Sciences, v.34, p.49-61, 2009.

CHOUDHARY, A.; AHI, J.. Biodiversity of Freshwater Insects: A Review. The International Journal of Engineering and Science, v.4, p.25-31, 2015.

COPATTI, C. E.. Diversidade de macroinvertebrados bentônicos na avaliação da qualidade ambiental de uma microbacia no sul do Brasil. Perspectiva, v.34, n.124, p.7991, 2010.

COSTA, E. B.. Distribuição de larvas de Chironominae (Insecta: Diptera: Chironomidae) em cursos d'água de baixa ordem sob influência de diferentes usos do solo no Município de Cabo Verde, MG. Dissertação (Mestrado em Ecologia e Tecnologia Ambiental) - Universidade Federal de Alfenas, Belo Horizonte, 2015.

CUMMINS, K. W.; KLUG, M. J.. Feeding ecology of stream invertebrates. Annual Reviews of Ecology. Evolution and Systematics, v.10, p.147-172, 1979.

ESTEVES, F. A.. Fundamentos de Limnologia. 3 ed. Rio de Janeiro: Interciência, 2011.

FERNÁNDEZ, H. R.; DOMINGUEZ, E.. Guía para la determinación de los artrópodos bentónicos sudamericanos. Tucumán: UNT, 2001.

FONTANA, L. E.; TONELLO, G.; RESTELLO, R. M.; HEPP, L. U.. A presença da agricultura altera a decomposição foliar e a composição das comunidades de invertebrados aquáticos em riachos subtropicais. Revista Perspectiva, v.44, n.165, p.7-20, 2020.

GIEHL, N. F.; RESENDE, B. O.; ROGES, P. F.; DIAS-SILVA, K.; NOGUEIRA, D. S.; CABETTE, H. S.. Diversidade de presas e predadores (Insecta) em mesohabitats de córregos de Cerrado. Iheringia, Série Zoologia, v.108, 2018.
HAMADA, N.; NESSIMIAN, J. L.; QUERINO, R. B.. Insetos aquáticos na Amazônia brasileira: taxonomia, biologia e ecologia. Manaus: INPA, 2014.

HENTGES, S. M.. Estrutura da comunidade de macroinvertebrados aquáticos e avaliação da qualidade da água em riachos da sub-bacia hidrográfica do rio Piratinim, Médio Rio Uruguai, RS, Brasil. Dissertação (Mestrado) Universidade Federal da Fronteira Sul, Chapecó, 2019.

HUGHES, A. O.; TANNER, C. C.; MCKERGOW, L. A.; SUKIAS, J. P. S.. Unrestricted dairy cattle grazing of a pastoral headwater wetland and its effect on water quality. Agric. Water Manag., v.165, p.72-81, 2016.

IBGE. Instituto Brasileiro de Geografia e Estatística. Rio Grande do Sul: Imigrante. IBGE, 2014.

IÑIGUEZ-ARMIJOS, C.; HAMPEL, H.; BREUER, L.. Land-use effects on structural and functional composition of benthic and leaf-associated macroinvertebrates in four Andean streams. Aquatic Ecology, v.52, n.1, p.77-92, 2018.

JOLY, C. A.; BICUDO, C. E. M.. Biodiversidade do Estado de São Paulo, Brasil: síntese do conhecimento ao final do século XX - 6: vertebrados. São Paulo: FAPESP, 1998.

KIKUCHI, R. M.; UIEDA, V. S.. Composição da comunidade de invertebrados de um ambiente lótico tropical e sua variação espacial e temporal. In: NESSIMIAN, J. L.; CARVALHO, A. L.. Ecologia de insetos aquáticos. Séries Oecologia Brasiliensis. Rio de Janeiro: PPGE-UFRJ, 1998. p.157-173.

KÖPPEN, W.. Climatologia. Ciudad de México: Fundo de Cultura Econômica, 1931.

LESZCZYŃSKA, J.; GRZYBKOWSKA, M.; GŁOWACKI, L.; DUKOWSKA, M.. Variables Influencing Chironomid Assemblages (Diptera: Chironomidae) in Lowland Rivers of Central Poland. Environmental Entomology, v.48, n.4, p.988-997, 2019.

LOPRETTO, E. C.; TELL, G.. Ecosistemas de aguas continentales: Metodologias para su estudio. La Plata: Ediciones Sur, 1995.

MARKUS, E.. Estrutura arbórea da mata ciliar como indicador ambiental em propriedades leiteiras com floresta estacional decidual. Dissertação (Mestrado em Ambiente e Desenvolvimento) - Centro Universitário UNIVATES, Lajeado, 2014.

MERRIT, R. W.; CUMMINS, K. W.. An introduction to the Aquatic Insects of North America. Dubuque: Kendal/Hunth Publishing Company, 1984.

MERRITT, R. W.; CUMMINS, K. W.; BERG, M. B.. Trophic relationships of macroinvertebrates. In Methods in Stream Ecology. Academic Press, v.1, p.413-433, 2017.

MUGNAI, R.; NESSIMIAN, J. L.; BAPTISTA, D. F.. Manual de identificação de macroinvertebrados aquáticos do estado do Rio de Janeiro. Rio de Janeiro: Technical Book, 2010.

NEGRÃO, G. N.; CUNHA, M. C.. Diversidade de 
macroinvertebrados bentônicos na avaliação do uso do solo e qualidade ambiental da bacia do Guabiroba, Guarapuava, PR, BrasiL. Revista Geografar, v.14, n.1, p.7-26, 2019.

NIH. Núcleo de Informações Hidrometeorológicas. Informações hidrometeorológicas. Lajeado: UNIVATES, 2012.

ONO, E. R.; MANOEL, P. S.; MELO, A. L. U.; UIEDA, V. S.. Effects of riparian vegetation removal on the functional feeding group structure of benthic macroinvertebrate assemblages. Community Ecology, p.145-157, 2020.

PES, A. M. O.; HAMADA, N.; NESSIMIAN, J. L.. Chaves de identificação de larvas para famílias e gêneros de Trichoptera (Insecta) da Amazônia Central, Brasil. Rev. Bras. de Entomologia, v.49, n.2, p.181-204, 2005.

PIO, J. F.; SANTIAGO, E. D. F.; COPATTI, C. E.. Composição e diversidade de macroinvertebrados bentônicos em um riacho do Cerrado brasileiro. Iheringia, Série Zoologia, v.110, 2020.

RIBEIRO, L. O.; UIEDA, V. S.. Estrutura da comunidade de macroinvertebrados bentônicos de um riacho de serra em Itatinga, São Paulo, Brasil. Rev. Bras. Zool., v.22, n.3, 2005.

RÍOS-TOUMA, B.; ENCALADA, A. C.; PRAT FORNELLS, N.. Macroinvertebrate assemblages of an andean high-altitude tropical stream: the importance of season and flow. Int. Rev. Hydrobiol., v.96, p.667-685, 2011.

SABATER, S.; BREGOLI, F.; ACUÑA, V.; BARCELÓ, D.; ELOSEGI, A.; GINEBREDA, A.; FERREIRA, V.. Effects of human-driven water stress on river ecosystems: a meta-analysis. Scientific Reports, v.8, n.1, p.1-11, 2018.

SCANES, C. G.. Chapter 18 - impact of agricultural animals on the environment. Anim. Hum. Soc., p.427-449, 2018.

SERRA, S. R. Q. GRAÇA, M.; DOLÉDEC, S.; FEIO, M. J..

Chironomidae traits and life history strategies as indicator of anthropogenic disturbance. Environmental Monitoring and Assesment, v.189, n.326, 2017. DOI: http://doi.org/10.1007/s10661-017-6027-y

SIEGLOCH, A. E.; SCHMITT, R.; SPIES, M. R.; PETRUCIO, M.; HERNÁNDEZ, M. I. M.. Effects of small changes in ripar-ian forest complexity on aquatic insect bioindicators in Brazilian subtropical streams. Marine \& Freshwater Research, v.68, p.519-527, 2016.

STROHSCHOEN, A. A. G.; PÉRICO, E.; LIMA, D. F. B.; REMPEL, C.. Estudo preliminar da qualidade da água dos rios Forqueta e Forquetinha, Rio grande do sul. Revista Brasileira de Biociências, v.7, n.4, p.372-375, 2009.

ZERWES, C. M.. Estrato arbóreo de fragmentos de floresta estacional decidual submontana em propriedades produtoras de leite: uso de dados fitossociológicos para diagnóstico de sustentabilidade. Dissertação (Mestrado em Ambiente e Desenvolviment) - Centro Universitário UNIVATES, Lajeado, 2015.

ZERWES, C. M.; REMPEL, C.; MORÁS, A. P. B.. Arboreal diversity in dairy-producing properties in the Arroio da Seca micro-river basin, Rio Grande do Sul, Brazil. Ciência e Natura, Santa Maria, v.40, n.e44, 2020.

A CBPC - Companhia Brasileira de Produção Científica (CNPJ: 11.221.422/0001-03) detém os direitos materiais desta publicação. Os direitos referem-se à publicação do trabalho em qualquer parte do mundo, incluindo os direitos às renovações, expansões e disseminações da contribuição, bem como outros direitos subsidiários. Todos os trabalhos publicados eletronicamente poderão posteriormente ser publicados em coletâneas impressas sob coordenação da Sustenere Publishing, da Companhia Brasileira de Produção Científica e seus parceiros autorizados. Os (as) autores (as) preservam os direitos autorais, mas não têm permissão para a publicação da contribuição em outro meio, impresso ou digital, em português ou em tradução. 